\title{
Self-motion direction discrimination in the visually impaired
}

\author{
${\text { Ivan } \text { Moser }^{1,2} \cdot \text { Luzia Grabherr }}^{3} \cdot$ Matthias Hartmann $^{1,2} \cdot$ Fred W. Mast $^{1,2}$
}

Received: 12 February 2015 / Accepted: 13 July 2015 / Published online: 31 July 2015

(C) Springer-Verlag Berlin Heidelberg 2015

\begin{abstract}
Despite the close interrelation between vestibular and visual processing (e.g., vestibulo-ocular reflex), surprisingly little is known about vestibular function in visually impaired people. In this study, we investigated thresholds of passive whole-body motion discrimination (leftward vs. rightward) in nine visually impaired participants and nine age-matched sighted controls. Participants were rotated in yaw, tilted in roll, and translated along the interaural axis at two different frequencies $(0.33$ and $2 \mathrm{~Hz})$ by means of a motion platform. Superior performance of visually impaired participants was found in the $0.33 \mathrm{~Hz}$ roll tilt condition. No differences were observed in the other motion conditions. Roll tilts stimulate the semicircular canals and otoliths simultaneously. The results could thus reflect a specific improvement in canal-otolith integration in the visually impaired and are consistent with the compensatory hypothesis, which implies that the visually impaired are able to compensate the absence of visual input.
\end{abstract}

Keywords Vestibular - Self-motion perception . Body motion $\cdot$ Psychophysics $\cdot$ Sensory threshold . Visual impairment

Ivan Moser

ivan.moser@psy.unibe.ch

1 Department of Psychology, University of Bern, Fabrikstrasse 8, Bern 3012, Switzerland

2 Center for Cognition, Learning and Memory, University of Bern, Fabrikstrasse 8, Bern 3012, Switzerland

3 Sansom Institute for Health Research, University of South Australia, GPO Box 2471, Adelaide, SA 5001, Australia

\section{Introduction}

It is a widespread assumption that blind people possess superior sensory abilities in non-visual modalities. In fact, they typically outperform sighted people in a wide range of auditory, tactile, and olfactory tasks (Collignon et al. 2006; Fieger et al. 2006; Goldreich and Kanics 2003; Gougoux et al. 2004; Hötting and Röder 2009; Rombaux et al. 2010; Stevens and Weaver 2005). However, other studies suggest a negative impact of visual deprivation on the remaining senses (Gori et al. 2010; Pasqualotto and Proulx 2012; Postma et al. 2008; Sterr et al. 2003; Zwiers et al. 2001). While much research in the domain of auditory, tactile, and olfactory perception has been carried out, substantially less is known about vestibular perception in visually impaired people. This is surprising given the fact that there is a close interrelation between vestibular and visual information processing. Visuo-vestibular interactions involve basic functions such as reflexive responses (e.g., vestibulo-ocular reflex) or the synchronization of circadian rhythms (Fuller et al. 2002; Martin et al. 2015), and they also play a role in higher-order cognitive functions such as spatial memory (Brandt et al. 2005; Schautzer et al. 2003) and mental imagery (Mast et al. 2006; Falconer and Mast 2012; Grabherr et al. 2011). A lack of visual experience can lead to abnormal development of these functions (Sack et al. 2000; Vecchi et al. 2004, but see Fortin et al. 2008). For example, the vestibulo-ocular reflex (VOR) is absent in congenitally blind people and present but reduced in people with acquired blindness (Kömpf and Piper 1987; Sherman and Keller 1986).

In the absence of visual references, the vestibular system is the predominant contributor to self-motion perception. Patients with vestibular loss show impaired self-motion perception in the dark (Cousins et al. 2013; Gianna et al. 
1996; Priesol et al. 2014; Valko et al. 2012). Seemungal et al. (2007) compared congenitally blind and sighted participants in three different self-motion perception tasks. After a sudden stop of constant yaw rotation on a rotating chair, the participants with congenital blindness showed shortened vestibular time constants (i.e., a faster decline of the perceived angular velocity of whole-body rotation), indicating a deficient velocity storage mechanism (Raphan et al. 1979). In the path-reversal task, participants needed to return as accurately as possible to the start position after being rotated in yaw. The path completion task required participants to return to the start position by completing the circle. Equal group performance was found in the pathreversal task. However, blind participants showed poorer performance in path completion. The authors explained this discrepancy by assuming different perceptual mechanisms for the two tasks. They argued that the path-reversal task can be accomplished by simply reproducing motion dynamics. In contrast, the path completion task requires an allocentric calibration of the vestibular-derived position, which was argued to be poorer in blind people, probably due to their use of less efficient non-allocentric strategies (Ungar et al. 1997).

Recently, we found that improvements in a self-motion perceptual training task were made only when visual information was present, highlighting the crucial role of visuovestibular interactions in self-motion perception (Hartmann et al. 2013). This finding suggests that the absence of a visuo-vestibular tuning in people with congenital blindness could lead to higher thresholds compared to sighted controls. However, it is also possible that visually impaired individuals are able to compensate the absence of visual information and thus outperform sighted controls. Our study might therefore add important evidence to the ongoing debate about the general loss hypothesis versus the compensatory hypothesis. The general loss hypothesis states that the lack or loss of a sensory modality is detrimental to the development of sensory or cognitive functioning. Adherents of the compensatory hypothesis, however, favor a view according to which sensory-deprived individuals are able to compensate for their lack of sensory input to some extent with remaining modalities. Resolving this discrepancy is not easy. Comparisons between groups (visually impaired people vs. sighted controls) do not yield robust results since they depend on task characteristics (for a comprehensive review, see Hötting and Röder 2009; Occelli et al. 2013). It is important to point out that visually impaired participants typically outperform sighted controls in tasks that require the discrimination of auditory, tactile, or olfactory stimuli (Alary et al. 2008; Collignon et al. 2006; Fieger et al. 2006; Gougoux et al. 2004; Hötting and Röder 2009; Rombaux et al. 2010; Stevens and Weaver 2005), whereas no consistent group differences are found in tasks that require mere detection of perceptual stimuli (Collignon et al. 2006; Niemeyer and Starlinger 1981; Schwenn et al. 2002; Starlinger and Niemeyer 1981; Yates et al. 1972).

In visually impaired participants and blindfolded sighted controls, we used psychophysical methods to assess self-motion direction discrimination thresholds using three different types of motion: yaw rotation around the earth-vertical axis (stimulation of the semicircular canals), interaural translation (stimulation of the otoliths), and a roll tilt rotation (combined canal-otolith stimulation). Testing possible dissociations between different types of motion is important. For example, Lewis et al. (2011b) found a specific hypersensitivity for roll tilts in patients with vestibular migraine compared to migraine patients without vertigo and healthy controls. This finding is consistent with the observed motion hypersensitivity and susceptibility to motion sickness in vestibular migraine patients (Dieterich and Brandt 1999; Lempert et al. 2012). Moreover, we recently found differences in self-motion perception thresholds between expert artistic gymnasts and controls exclusively for interaural translations (Hartmann et al. 2014). These results show that differences in performance between two groups can be selective, depending on the specific vestibular stimulation elicited by a particular type of motion.

In addition to this, we measured thresholds at two different frequencies because vestibular thresholds depend on frequency (Grabherr et al. 2008; Valko et al. 2012). Importantly, Karmali et al. (2014), who directly compared visual and vestibular motion perception, found lower visual thresholds at frequencies between 0.1 and $1 \mathrm{~Hz}$ and observed the opposite pattern at frequencies higher than $2 \mathrm{~Hz}$. If visual motion perception outweighs vestibular motion perception at lower frequencies, the compensatory hypothesis suggests superior performance of the visually impaired at low frequencies. The absence of visual input disrupts performance of sighted people when compared to the visually impaired who are used to perceive self-motion without visual input. In contrast, the general loss hypothesis suggests worse performance in the visually impaired for both frequencies.

\section{Methods}

\section{Participants}

Ten visually impaired participants (six females, one lefthanded), aged 20-60 years (mean age $=37.2$ ) were recruited through a self-help organization of blind and visually impaired people in Switzerland and a radio station for visually impaired people. Nine of ten respondents were 
Table 1 Visually impaired participants' characteristics

\begin{tabular}{lllll}
\hline Participant & Sex & Age (years) & $\begin{array}{l}\text { Residual } \\
\text { vision }(\%)^{\mathrm{a}}\end{array}$ & $\begin{array}{l}\text { Onset of blindness } \\
\text { (years) }\end{array}$ \\
\hline B1 & M & 27 & 2 & Birth \\
B2 & M & 31 & 10 & Birth \\
B3 & W & 23 & 2 & 5 \\
B4 & M & 23 & 0 & 11 \\
B5 & W & 48 & 0 & 30 \\
B6 & W & 20 & 4 & Birth \\
B7 & W & 48 & 3 & 16 \\
B8 & M & 60 & 0 & Birth \\
B9 & W & 34 & 0 & Birth \\
\hline
\end{tabular}

a Numbers in percent indicate the amount of residual visual acuity

blind according to the definition of the World Health Organization (2013) and the International Council of Ophthalmology (2002). ${ }^{1}$ One male participant reported having a residual vision of $10 \%$. His visual acuity was considered as severe visual impairment according to the WHO definition, and the participant remained in the experimental group. Six participants had congenital impairments, while four had lost their vision progressively during childhood, adolescence, or adulthood (see Table 1 for more detailed participants' characteristics).

The participants completed a questionnaire to indicate handedness, education, profession, sport activities, as well as general health and possible medication. One female participant reported severe postural vertigo in daily life situations with pending diagnosis and was therefore excluded from the final dataset. None of the remaining nine participants (five females, mean age $=34.9, \mathrm{SD}=14.0$ ) reported any history of neurological, vestibular, or attentional disorders. One participant was taking antidepressant medication.

Nine healthy participants (five females, one lefthanded) with normal or corrected-to-normal vision, aged 20-52 years (mean age $=32.8, \mathrm{SD}=11.2$ ) participated as gender- and age-matched controls. None of the participants reported any history of neurological, vestibular, or attentional disorders.

All participants gave informed consent prior to the experiment. The participants received information about the study including the informed consent form by email. All visually impaired participants confirmed that they were able to read this electronic document in advance with the help of their personal screen reader software. The study was approved by the local ethics committee, and testing

\footnotetext{
1 "Blindness" is defined as a visual acuity $<5 \%$ or a visual acuity worse than $3 / 60$ on the Snellen chart, respectively. A visual acuity between $3 / 60$ and $6 / 60$ is referred to as "severe visual impairment" (World Health Organization 2013).
}

was performed according to the ethical standards defined by the Declaration of Helsinki.

\section{Apparatus}

Participants were seated and fastened with a five-point harness in a car seat, which was mounted on a MOOG motion platform (6DOF2000E, Moog Inc., NY; see Fig. 1 in Hartmann et al. (2012) for an image of the apparatus). An adjustable helmet fixed to the back of the seat was used to ensure a stable and upright head position. Additionally, pink noise (at approximately $60 \mathrm{~dB}$ ) was delivered through in-ear headphones to mask auditory cues originating from the motion platform. The participants' eyes were blindfolded, and the experiment was conducted in a dark room.

\section{Motion stimuli}

Three different types of motion were applied, which all required rightward-leftward discrimination. Participants were (1) rotated in yaw, (2) tilted in roll, and (3) translated along the interaural axis. As in previous experiments (Benson et al. 1989; Grabherr et al. 2008), motion profiles consisted of single cycles of sinusoidal acceleration $[a(t)=A$ $\sin (2 \pi f t)]$, where $A$ is the acceleration amplitude and $f$ is the frequency using this sort of motion profile and beginning the motion at zero velocity, peak velocity $\left(v_{\max }=A T / \pi\right)$ and the total lateral displacement $\left(\Delta p=A T^{2} / 2 \pi\right)$ are proportional to the peak acceleration $(A)$. Each type of motion was tested at durations of 3 and $0.5 \mathrm{~s}$, which equal frequencies of 0.33 and $2 \mathrm{~Hz}$ as their inverse $(T=1 / f)$.

\section{Procedure}

Seated on the motion platform, the participants held a button in each hand and were instructed to press the button in their left hand when feeling a leftward motion and to press the button in their right hand when feeling a rightward motion, respectively. A low-pitch sound, played $2000 \mathrm{~ms}$ before motion stimulus onset, prepared the participants for motion onset. Five hundred milliseconds after each motion, a high-pitch tone indicated that a response needed to be given. Participants were instructed to make their best guess when they were unsure about the direction of the motion. After the response was given, the motion direction of the next trial was randomly triggered with a delay of approximately $3 \mathrm{~s}$. If necessary, the experimenter reversed the direction manually to avoid reaching the displacement limits of the device. Overall, including the duration of the offset and onset motion tone and the participant's reaction time, two motions were separated with a delay of at least $6.5 \mathrm{~s}$. 
The order of the six experimental blocks (yaw rotation, roll rotation, and interaural translation at 0.33 and $2 \mathrm{~Hz}$ each) was counterbalanced across participants. The starting velocity values for the first trial of each block were chosen well above thresholds. For the high frequencies $(2 \mathrm{~Hz})$, the starting values were set at $5 \%$ for yaw, $5 \%$ for roll, and $3 \mathrm{~cm} / \mathrm{s}$ for interaural translation. The starting values for the low frequencies $(0.33 \mathrm{~Hz})$ were $8 \%$ for yaw, $7 \%$ s for roll, and $6 \mathrm{~cm} / \mathrm{s}$ for interaural translation. After each block, a short break was provided.

Thresholds were determined using a three-down, one-up PEST staircase paradigm (Leek 2001; Merfeld 2011; Taylor and Creelman 1967). This adaptive method requires the participants to correctly identify three subsequent movements in order for the velocity level to be reduced. Any false response results in an increase in the velocity level. The procedure targets a threshold at which $79.4 \%$ of trials are correctly identified. This threshold definition is a direct consequence of the adaptive threedown, one-up staircase. In this paradigm, the stimulus level varies around an asymptote at which increases and decreases occur with equal probability. Thus, at the staircase asymptote, the probability ( $p$ ) of providing an incorrect answer after three correct answers equals the probability of providing three correct answers in a row $\left(p^{3}=0.5\right)$. Consequently, the probability of a single correct answer equals the cube root of 0.5 , which is 0.794 (Leek 2001; Merfeld 2011).

The staircase was completed when the number of four minimum and four maximum reversals was reached. ${ }^{2}$ Using this staircase procedure, one block lasted 55 trials on average (range 29-90 trials). Thresholds were computed by means of a psychometric curve fit run on MATLAB using the statistics toolbox (version 8.2). For each motion type, a Gaussian cumulative distribution function was fit to the threshold data using a generalized linear model (Haburcakova et al. 2012; Karmali et al. 2014; MacNeilage et al. 2010; Soyka et al. 2011; Valko et al. 2012). The Gaussian distribution function is described by an offset from zero $(\mu)$ and the standard deviation of the noise $(\sigma)$. It was fit to the data with a probit link function, yielding a maximum likelihood fit (Haburcakova et al. 2012).

\section{Data analysis}

Repeated-measures analysis of variance (ANOVA) with the between-subject variable group (visually impaired

\footnotetext{
${ }^{2}$ A minimum reversal occurs when a participant provides the wrong answer and the stimulus level increases accordingly. A maximum reversal occurs when the subject correctly detects three subsequent motion stimuli after having made an error on the previous trial. See Figure 3 in Hartmann et al. (2014) for an example of a procedure.
}

participants vs. sighted controls) and the within-subject variable frequency $(0.33$ vs. $2 \mathrm{~Hz})$ was conducted for each type of motion (yaw, roll, and interaural translation). Velocity thresholds were (measured in $\%$ s for yaw rotation and roll tilt and $\mathrm{cm} / \mathrm{s}$ for interaural translation) the dependent variable. Log-transformed thresholds did not violate the assumption of normality $(p>0.2)$ except for high-frequency interaural discrimination $(p<0.001)$. A qualitative assessment of the data revealed that two participants showed a substantial increase in threshold levels in the middle of the high-frequency interaural translation recording session. Fitting a generalized linear model resulted in a large overestimation of threshold levels, and we decided to use the simple but established "last minimum last maximum" method for this type of motion. Accordingly, interaural discrimination thresholds were obtained by averaging the peak velocity levels of the last two direction reversals (Grabherr et al. 2008; Hartmann et al. 2014). The data analysis was performed on log-transformed values. The mean thresholds were subsequently back-transformed to physical units for better understanding.

\section{Results}

The back-transformed mean thresholds for all types of motion are listed in Table 2 and illustrated in Fig. 1a (low frequency $0.33 \mathrm{~Hz}$ ) and Fig. 1b (high frequency $2 \mathrm{~Hz}$ ). Upper and lower limits of the back-transformed $95 \%$ confidence intervals are reported instead of standard deviations since the latter cannot be transformed back to the original scale (Bland and Altman 1996). The mean thresholds and their large interindividual variability are comparable to similar studies of self-motion direction discrimination in healthy people (Benson et al. 1989; Grabherr et al. 2008; Hartmann et al. 2014; Karmali et al. 2014; Valko et al. 2012). Results from the repeated-measures ANOVA are summarized for each type of motion below.

\section{Yaw rotation}

There was neither a main effect of group, $F(1,16)=0.507$, $p=0.49, \eta_{p}^{2}=.03$, nor an interaction between group and frequency, $F(1,16)=0.04, p=0.85, \eta_{p}^{2}<.01$. The visually impaired participants $(M=0.81,95 \% \mathrm{CI}[0.61,1.08])$ showed no difference in mean discrimination thresholds compared to the sighted controls $(M=0.94,95 \%$ CI [0.65, 1.34]). As expected, there was a significant main effect of frequency, $F(1,16)=4.76, p=0.04, \eta_{p}^{2}=.23$. Thresholds for the high-frequency condition $(M=0.62,95 \%$ CI $[0.52$, $0.74])$ were lower than those of the low-frequency condition $(M=1.10,95 \% \mathrm{CI}[0.77,1.57])$. 
Table 2 Mean velocity $\left(v_{\max }\right)$ and displacement $(\Delta p)$ thresholds ${ }^{\mathrm{a}}$ for visually impaired participants (VIP) and sighted controls (SC) in all motion conditions

\begin{tabular}{|c|c|c|c|c|}
\hline & \multicolumn{2}{|l|}{$0.33 \mathrm{~Hz}$} & \multicolumn{2}{|l|}{$2 \mathrm{~Hz}$} \\
\hline & VIP $[95 \% \mathrm{CI}]$ & $\mathrm{SC}[95 \% \mathrm{CI}]$ & VIP $[95 \% \mathrm{CI}]$ & $\mathrm{SC}[95 \% \mathrm{CI}]$ \\
\hline \multicolumn{5}{|c|}{ Yaw rotation } \\
\hline$v_{\max }$ & $1.00[0.63,1.58]$ & $1.20[0.63,2.30]$ & $0.69[0.54,0.88]$ & $0.73[0.50,1.06]$ \\
\hline$\Delta p$ & $1.50[0.95,2.37]$ & $1.80[0.94,3.46]$ & $0.17[0.14,0.22]$ & $0.18[0.12,0.27]$ \\
\hline \multicolumn{5}{|c|}{ Roll tilt } \\
\hline$v_{\max }$ & $0.36[0.24,0.56]$ & $0.67[0.47,0.96]$ & $0.22[0.19,0.26]$ & $0.22\left[\begin{array}{lll}0.16 & 0.29\end{array}\right]$ \\
\hline$\Delta p$ & $0.54[0.35,0.84]$ & $1.01[0.71,1.44]$ & $0.06[0.05,0.06]$ & $0.06[0.04,0.07]$ \\
\hline \multicolumn{5}{|c|}{ Interaural translation } \\
\hline$v_{\max }$ & $2.17[1.35,3.50]$ & $2.39[1.40,4.06]$ & $0.30[0.22,0.42]$ & $0.40[0.30,0.52]$ \\
\hline$\Delta p$ & $3.26[2.02,5.25]$ & $3.59[2.11,6.10]$ & $0.08[0.05,0.10]$ & $0.10[0.08,0.13]$ \\
\hline
\end{tabular}

${ }^{\text {a }}$ All thresholds are expressed in back-transformed physical units. Peak velocity thresholds $\left(v_{\max }\right)$ are reported in $\% \mathrm{~s}$ for angular motion (yaw rotation, roll tilt) and $\mathrm{cm} / \mathrm{s}$ for interaural translation. Displacement thresholds $(\Delta p)$ are reported in ${ }^{\circ}$ for angular motion and $\mathrm{cm}$ for interaural translation

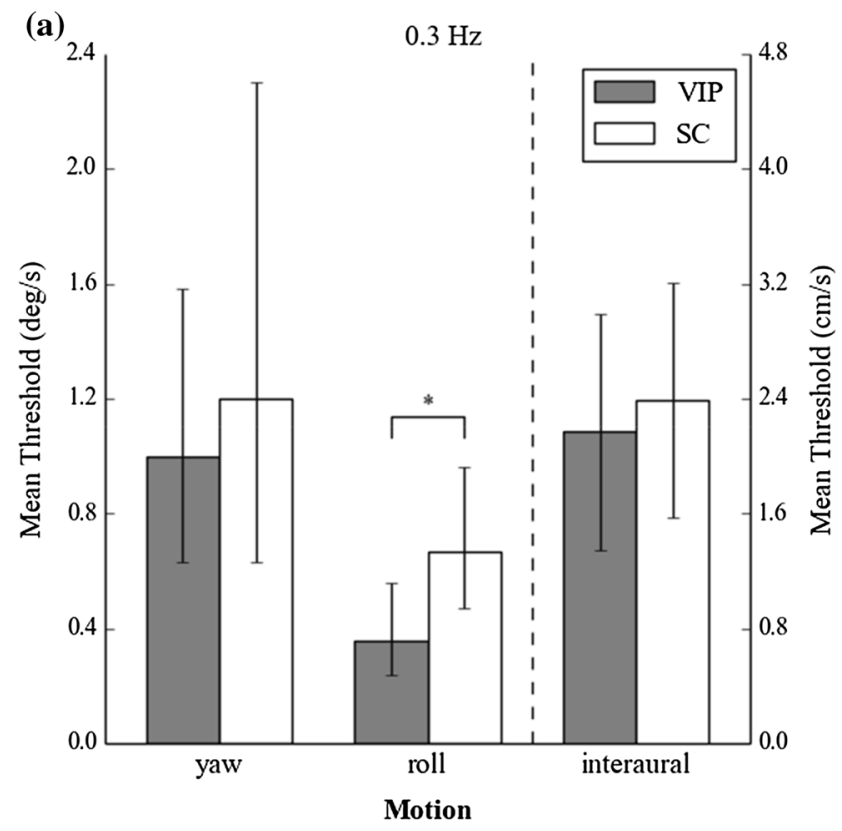

Fig. 1 Mean direction discrimination thresholds of passive selfmotion for a low $(0.33 \mathrm{~Hz})$ and $\mathbf{b}$ high frequency $(2 \mathrm{~Hz})$. Error bars represent $95 \%$ confidence intervals. Asterisks indicate significant dif-

\section{Roll rotation}

A trend toward a significant main effect was observed between the visually impaired and the sighted controls, $F(1,16)=3.20, p=0.09, \eta_{p}^{2}=.17$. Mean thresholds tended to be lower in the visually impaired participants $(M=0.29,95 \%$ CI $[0.22,0.36])$ when compared to the sighted controls $(M=0.38,95 \%$ CI $[0.27,0.55])$. There was a significant interaction between group and frequency, $F(1,16)=7.83, p=0.01, \eta_{p}^{2}=.33$. In the low-frequency

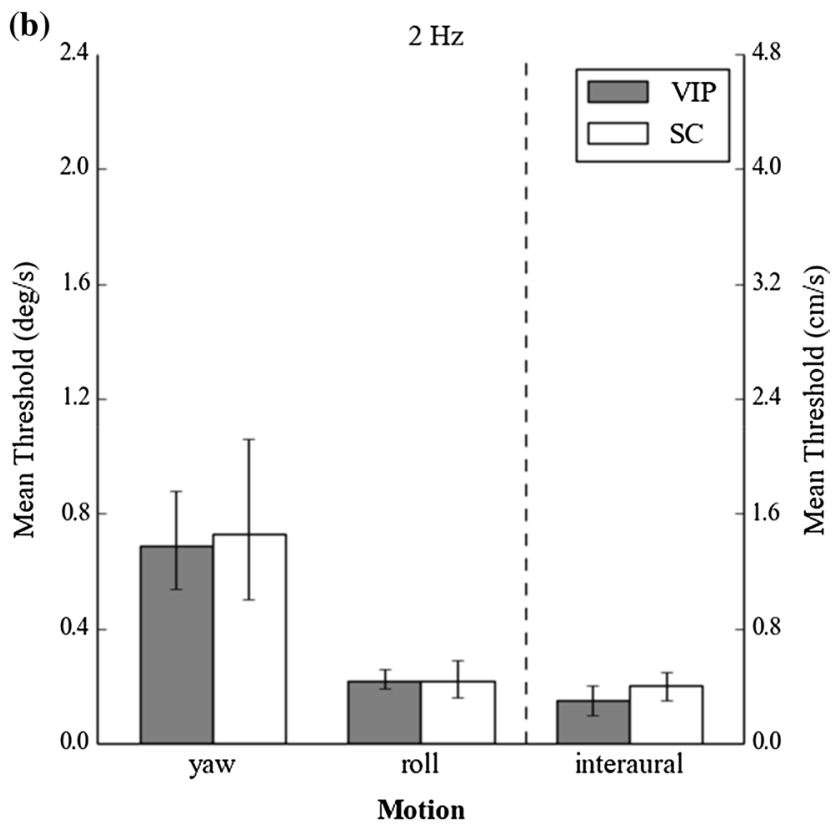

ferences $(p<0.05)$ between visually impaired participants (VIP) and sighted controls (SC)

condition, Bonferroni post hoc tests revealed significantly lower thresholds $(p=0.02)$ for the visually impaired $(M=0.36,95 \%$ CI $[0.23,0.56])$ when compared to the sighted controls $(M=0.67,95 \% \mathrm{CI}[0.47,0.96])$. In contrast, there was no difference in the high-frequency condition $(p=0.80)$ between the visually impaired $(M=0.22$, $95 \%$ CI $[0.19,0.26])$ and sighted participants $(M=0.22$, $95 \%$ CI $[0.16,0.29])$. Furthermore, there was a significant main effect of frequency, $F(1,16)=47.89, p<0.001$, $\eta_{p}^{2}=.75$, with lower mean thresholds in the high-frequency 
condition $(M=0.18,95 \% \mathrm{CI}[0.16,21])$ than in the lowfrequency condition $(M=0.50,95 \% \mathrm{CI}[0.37,0.67])$.

\section{Interaural translation}

No main effect of group, $F(1,16)=0.98, p=0.34$, $\eta_{p}^{2}=.06$, was observed, and frequency did not interact with group either, $F(1,16)=0.26, p=0.62, \eta_{p}^{2}=.02$. Thresholds of the visually impaired participants $(M=0.81$, $95 \%$ CI $[0.46,1.43])$ did not differ from the sighted controls $(M=0.97,95 \%$ CI $[0.57,1.65])$. As for yaw and roll rotation, there was a highly significant main effect of frequency, $F(1,16)=119.16, p<0.001, \eta_{p}^{2}=.88$. Thresholds for the high-frequency condition $(M=0.35,95 \%$ CI [0.28, $0.42])$ were lower than those for the low-frequency condition $(M=2.28,95 \%$ CI $[1.66,3.13])$.

\section{Additional analyses}

Due to the small sample size, we also analyzed our data by means of distribution-free two-sample permutation tests (Good 2011; Hesterberg et al. 2005) in order to critically evaluate the observed group difference in the lowfrequency roll tilt condition. In a first step, (1) we calculated the observed difference in means between the visually impaired and the sighted group $\left(M_{\mathrm{VIP}-S C}\right)$. Under the null hypothesis, there is no difference between the two groups, which leads to the exchangeability of group labels. Given this assumption, (2) group labels (visually impaired vs. sighted) were shuffled 500,000 times and each time the mean difference was calculated. This procedure resulted in the sampling distribution of the shuffled mean differences under the null hypothesis. Finally, (3) the proportion of shuffled mean differences greater (ignoring sign) or equal than the observed difference in (1) yielded the $p$ value. In line with results observed with the repeated-measures ANOVA, the two-sample permutation test showed a significant group difference in the low-frequency roll tilt condition. Visually impaired participants showed lower discrimination thresholds than sighted controls $\left(M_{\mathrm{VIP}-\mathrm{SC}}=-0.24\right.$, $p=0.04$ ).

To examine the possible influence of visual experience on threshold levels, the visually impaired participants were split into a congenitally blind group $(n=5)$ and another group with acquired blindness $(n=4)$. Facing small sample sizes, the data were analyzed only by means of twosample permutation tests (and not ANOVAs) for each type of motion. None of the motion conditions showed significant group differences between congenitally blind participants and participants with acquired blindness $(p \geq 0.27$ for all types of motion).

\section{Discussion}

The aim of the present study was to examine whether vestibular substitution occurs as a consequence of visual deprivation in the form of lower direction discrimination thresholds in self-motion perception. Interestingly, superior performance of the visually impaired participants was found when participants were rotated in roll at a frequency of $0.33 \mathrm{~Hz}$. The comparison of direction discrimination thresholds revealed no differences between visually impaired and sighted participants for yaw rotation and interaural translation.

We argue that the divergent findings between roll tilts and the two remaining types of motion can be explained by the fact that roll tilts differ from the other motion types in one important aspect. Yaw rotation and interaural translation lead to an isolated stimulation of the semicircular canals and the otolith organs, respectively. In contrast, roll tilts involve the simultaneous activation of the semicircular canals and otoliths since they entail an angular displacement that also changes the head position in relation to gravity. This difference is also reflected in quantitative observations of the VOR. Higher VOR gain was reported for off-vertical roll and pitch rotations (combined canalotolith stimulation) compared to gravity-neutral roll and pitch rotations that stimulate the canals only (Bockisch et al. 2005). If the VOR and self-motion perception are tightly connected (Bertolini et al. 2011; Bronstein et al. 2008; Okada et al. 1999, but see Haburcakova et al. 2012), it suggests that self-motion discrimination in the roll plane differs from self-motion discrimination for yaw rotations and interaural translation.

The qualitative difference of roll tilts is further supported by findings from vestibular migraine patients. Lewis et al. (2011b), who compared self-motion thresholds in patients with vestibular migraine, migraine patients with no history of vertigo and healthy controls, report striking parallels to our results. They observed a specific hypersensitivity in patients with vestibular migraine for roll tilts (stimulating the canals and otoliths simultaneously). At the same time, they found no reduction in motion thresholds when the participants were rotated in roll in a supine position (stimulating the semicircular canals only) and very slow roll tilts (stimulating the otoliths only). Lewis et al. (2011b) suggest that their results reflect abnormal canal-otolith integration in patients with vestibular migraine. They argue that altered signal processing in Purkinje cells of the caudal cerebellar vermis might explain the specific changes observed in roll tilts; these neurons are involved in canal-otolith integration (Angelaki et al. 2010; Wearne et al. 1998). Interestingly, blind people also show abnormalities in cerebellar 
signal processing. It has been shown that the density of benzodiazepine receptors in the blind is reduced compared to sighted controls. Together with cerebral reorganization, the reduction in benzodiazepine receptors in the cerebellum might contribute to the sensory compensation processes in the blind in terms of lower neuronal thresholds (SanabriaBohorquez et al. 2001). One could even go a step further and speculate that the downregulation of benzodiazepine receptors leads to lower neuronal thresholds for the integrated canal-otolith cues reaching the cerebellum. Hypothetically, such a process could explain the specific reduction in roll tilt thresholds in visually impaired participants. However, it is also possible that the specific improvement in roll tilts might reflect a change of strategy through the recruitment of other neuronal networks. For example, such a mechanism has been reported in animal research (rats) showing a shift from a hippocampal to a striatal memory strategy after vestibular lesions (Machado et al. 2014). Similarly, an upregulation of visuo-proprioceptive interactions has been observed as a consequence of bilateral vestibular loss in humans (Cutfield et al. 2014). Since there is a large overlap of visual, vestibular, and proprioceptive signal processing at the cortical level (Akbarian et al. 1992; Brandt and Dieterich 1999; Cutfield et al. 2014; Guldin et al. 1992), it is conceivable that visual deprivation affects the integration of the remaining sensory signals. More specifically, upregulated sensitivity to extra-vestibular cues such as somatosensory graviceptive input (Mittelstaedt 1996) could explain the observed group difference for roll tilts. This type of motion changed the participants' head orientation with respect to gravity, which provided a sustained change in somatosensory cues after the vestibular stimulation elicited by the motion platform. In contrast, potential somatosensory cues were only transient in interaural translation and yaw rotation (Seemungal et al. 2004). Interestingly, patients with bilateral vestibular loss perform much worse in yaw rotation, interaural, and vertical translation, but close to normal for roll tilt discrimination (Bisdorff et al. 1996; Valko et al. 2012). Thus, it is by all means possible that the visually impaired are more able than sighted people to use extra-vestibular somatosensory cues to infer motion direction. To date, however, there is no consensus whether blind people show superior somatosensory performance (Alary et al. 2009).

In contrast to roll tilts, we did not observe group differences in the other types of motion. However, it has to be pointed out that the lack of behavioral group differences does not exclude the possibility of increased sensitivity on the cortical level. In fact, a large body of research has shown that the visually impaired exhibit improved topdown modulation and substantial reorganization across different brain regions even in the absence of behavioral differences (Occelli et al. 2013). For example, Niemeyer and Starlinger (1981) found no differences in auditory thresholds but reported shortened N1 latencies in the EEG signal for blind participants, which is thought to reflect improved auditory information processing. In the same manner, it is conceivable that central compensation mechanisms are also present in the processing of gravity-neutral types of motion.

Intriguingly, we only found group differences for lowfrequency roll tilts as opposed to the absence of group differences for high-frequency roll tilts. We argue that this selective group difference is in line with the findings of Karmali et al. (2014), who showed that visual motion perception is more precise than vestibular motion perception at low frequencies (between 0.1 and $1 \mathrm{~Hz}$ ). If sighted people strongly rely on visual cues at these frequencies, they are expected to perform worse than the visually impaired in the absence of visual cues, since the latter are already used to perceive self-motion without visual input. Consistent with this prediction, we found superior performance of the visually impaired for low-frequency roll tilts only. In contrast, the group difference was absent for high-frequency roll tilts, where also sighted people primarily rely on vestibular motion perception (Karmali et al. 2014). Moreover, the discrepancy of our findings between low- and high-frequency roll tilts might be explained by frequency-dependent contributions of the semicircular canals and the otoliths to self-motion perception. At $0.3 \mathrm{~Hz}$, self-motion perception thresholds are thought to depend on combined canal-otolith signals, whereas canal cues dominate self-motion perception at higher frequencies and above $1 \mathrm{~Hz}$ (King et al. 2014; Lewis et al. 2011a, b). This line of evidence further corroborates that the visually impaired show a selective improvement in canal-otolith integration.

The fact that visually impaired individuals did not show an impaired performance in self-motion discrimination when compared to controls (and even a superior performance in the case of roll tilt) demonstrates that the loss of vision did not lead to a general impairment of vestibular perception. While visuo-vestibular interaction seems to be a critical factor for learning to discriminate self-motion direction in sighted individuals (Hartmann et al. 2013), visually impaired individuals are able to develop strategies to compensate for the lack of visuo-vestibular interactions. Thus, our results are not in line with the general loss hypothesis but rather support the compensatory hypothesis. As pointed out above, such compensation could involve an increased vestibular sensitivity or/and upregulated integration of signals stimulating the remaining sensory organs.

In the case of roll tilts, increased sensitivity in the blind might be relevant from a functional point of view. Since roll tilts entail changes in gravitational force, these off-vertical 
angular displacements are more likely to be associated with falls. Blind people may be more sensitive to register relevant cues that provide information about possible falls. In fact, blind people have demonstrated poorer balance (Ray et al. 2008) and a more cautious walking strategy (Hallemans et al. 2010) and might therefore compensate their higher risk of falling with increased specific sensitivity to corresponding movements.

As an important limitation, however, we would like to point out that our sample of visually impaired participants was heterogeneous regarding their visual experience. Indeed, earlier studies have shown differences in VOR between congenital and acquired blindness (Kömpf and Piper 1987; Sherman and Keller 1986). However, it is still not clear how the VOR relates to self-motion perception (Bertolini et al. 2011; Bronstein et al. 2008; Haburcakova et al. 2012; Okada et al. 1999). Moreover, this limitation can be somewhat mitigated since many studies failed to demonstrate group differences between early- and late-blind individuals, and some studies report above-normal performance for both subgroups (Fieger et al. 2006; Goldreich and Kanics 2003; Röder and Rösler 2003; Schwenn et al. 2002; Voss et al. 2004). In line with these findings, an additional analysis of our data showed no differences between early-blind participants and participants with acquired blindness. Nevertheless, the influence of visual experience is still debated as other studies have shown superior performance selectively for early-blind participants (Gougoux et al. 2004; Röder et al. 2004; Wan et al. 2010). It is unclear whether age at blindness onset affects self-motion perception, and in this regard, our results can only be considered as preliminary. Ideally, further studies will need to compare larger subsamples of early- and late-blind individuals and try to disentangle the role of age at blindness onset and duration of blindness. Moreover, future studies should measure roll thresholds in blind participants across different methods (see Lewis et al. 2011a). A suitable approach would be to compare roll tilts (combined canal-otolith activation) with supine roll rotation (pure canal activation) and quasi-static roll tilts (tilting the participants at very low velocities which mainly induces otolith activation). Using this approach, it would be possible to determine the relative influence of angular and translational components of roll tilts and quantify the beneficial effect of combined canal-otolith to self-motion thresholds of visually impaired people.

Taken together, visually impaired participants showed superior performance to discriminate roll tilt direction. Upregulated canal-otolith integration and/or integration of extra-vestibular cues may explain this result. There were no differences in self-motion direction discrimination for yaw rotation and interaural translation. The results are consistent with the compensatory hypothesis, which implies that the visually impaired are able to compensate the absence of visual input with their remaining senses.

Acknowledgments We would like to thank Vanda Lory for technical assistance and both Patrick Kilchör and Katharina Engelhart for help in recruiting visually impaired participants. This research was supported by the Swiss National Science Foundation (Sinergia Grant "Balancing Self and Body", CRSII1_125135) and a postdoctoral fellowship awarded to Luzia Grabherr.

\section{References}

Akbarian S, Grusser OJ, Guldin WO (1992) Thalamic connections of the vestibular cortical fields in the squirrel-monkey (Saimiri-sciureus). J Comp Neurol 326:423-441. doi:10.1002/ Cne.903260308

Alary F, Goldstein R, Duquette M, Chapman CE, Voss P, Lepore F (2008) Tactile acuity in the blind: a psychophysical study using a two-dimensional angle discrimination task. Exp Brain Res 187:587-594. doi:10.1007/S00221-008-1327-7

Alary F, Duquette M, Goldstein R, Chapman CE, Voss P, La Buissonniere-Ariza V, Lepore F (2009) Tactile acuity in the blind: a closer look reveals superiority over the sighted in some but not all cutaneous tasks. Neuropsychologia 47:2037-2043. doi:10.1016/J.Neuropsychologia.2009.03.014

Angelaki DE, Yakusheva TA, Green AM, Dickman JD, Blazquez PM (2010) Computation of egomotion in the macaque cerebellar vermis. Cerebellum 9:174-182. doi:10.1007/s12311-009-0147-z

Benson AJ, Hutt ECB, Brown SF (1989) Thresholds for the perception of whole-body angular movement about a vertical axis. Aviat Space Environ Med 60:205-213

Bertolini G, Ramat S, Laurens J, Bockisch CJ, Marti S, Straumann D, Palla A (2011) Velocity storage contribution to vestibular selfmotion perception in healthy human subjects. J Neurophysiol 105:209-223. doi:10.1152/Jn.00154.2010

Bisdorff AR, Wolsley CJ, Anastasopoulos D, Bronstein AM, Gresty MA (1996) The perception of body verticality (subjective postural vertical) in peripheral and central vestibular disorders. Brain 119:1523-1534. doi:10.1093/Brain/119.5.1523

Bland JM, Altman DG (1996) Transformations, means, and confidence intervals. $\mathrm{Br}$ Med J 312:1079. doi:10.1136/ bmj.312.7038.1079

Bockisch C, Straumann D, Haslwanter T (2005) Human 3-D aVOR with and without otolith stimulation. Exp Brain Res 161:358367. doi:10.1007/s00221-004-2080-1

Brandt T, Dieterich M (1999) The vestibular cortex-its locations, functions, and disorders. Ann NY Acad Sci 871:293-312. doi:10.1111/J.1749-6632.1999.Tb09193.X

Brandt $\mathrm{T}$ et al (2005) Vestibular loss causes hippocampal atrophy and impaired spatial memory in humans. Brain 128:2732-2741. doi:10.1093/brain/awh617

Bronstein AM, Grunfeld EA, Faldon M, Okada T (2008) Reduced self-motion perception in patients with midline cerebellar lesions. Neuroreport 19:691-693

Collignon O, Renier L, Bruyer R, Tranduy D, Veraart C (2006) Improved selective and divided spatial attention in early blind subjects. Brain Res 1075:175-182. doi:10.1016/J. Brainres.2005.12.079

Cousins S et al (2013) Vestibular perception following acute unilateral vestibular lesions. PLoS One. doi:10.1371/journal.pone.0061862

Cutfield NJ, Scott G, Waldman AD, Sharp DJ, Bronstein AM (2014) Visual and proprioceptive interaction in patients with bilateral 
vestibular loss. Neuroimage Clin 4:274-282. doi:10.1016/j. nicl.2013.12.01

Dieterich M, Brandt T (1999) Episodic vertigo related to migraine (90 cases): vestibular migraine? J Neurol 246:883-892

Falconer CJ, Mast FW (2012) Balancing the mind. Exp Psychol 59:332-339. doi:10.1027/1618-3169/a000161

Fieger A, Roder B, Teder-Salejarvi W, Hillyard SA, Neville HJ (2006) Auditory spatial tuning in late-onset blindness in humans. Cogn Neurosci 18:149-157. doi:10.1162/089892906775783697

Fortin M et al (2008) Wayfinding in the blind: larger hippocampal volume and supranormal spatial navigation. Brain 131:29953005. doi:10.1093/Brain/Awn250

Fuller PM, Jones TA, Jones SM, Fuller CA (2002) Neurovestibular modulation of circadian and homeostatic regulation: vestibulohypothalamic connection? Proc Natl Acad Sci USA 99:1572315728. doi:10.1073/Pnas.242251499

Gianna C, Heimbrand S, Gresty M (1996) Thresholds for detection of motion direction during passive lateral whole-body acceleration in normal subjects and patients with bilateral loss of labyrinthine function. Brain Res Bull 40:443-447. doi:10.1016/0361-9230(96)00140-2

Goldreich D, Kanics IM (2003) Tactile acuity is enhanced in blindness. J Neurosci 23:3439-3445

Good P (2011) A practitioner's guide to resampling for data analysis, data mining, and modeling. Chapman \& Hall/CRC

Gori M, Sandini G, Martinoli C, Burr D (2010) Poor haptic orientation discrimination in nonsighted children may reflect disruption of cross-sensory calibration. Curr Biol 20:223-225. doi:10.1016/J.Cub.2009.11.069

Gougoux F, Lepore F, Lassonde M, Voss P, Zatorre RJ, Belin P (2004) Pitch discrimination in the early blind. Nature 430:309. doi:10.1038/430309a

Grabherr L, Nicoucar K, Mast FW, Merfeld DM (2008) Vestibular thresholds for yaw rotation about an earth-vertical axis as a function of frequency. Exp Brain Res 186:677-681. doi:10.1007/ s00221-008-1350-8

Grabherr L, Cuffel C, Guyot J-P, Mast FW (2011) Mental transformation abilities in patients with unilateral and bilateral vestibular loss. Exp Brain Res 209:205-214. doi:10.1007/ s00221-011-2535-0

Guldin WO, Akbarian S, Grusser OJ (1992) Corticocortical connections and cytoarchitectonics of the primate vestibular cortex-a study in squirrel-monkeys (saimiri-sciureus). J Comp Neurol 326:375-401. doi:10.1002/Cne.903260306

Haburcakova C, Lewis RF, Merfeld DM (2012) Frequency dependence of vestibuloocular reflex thresholds. J Neurophysiol 107:973-983. doi:10.1152/Jn.00451.2011

Hallemans A, Ortibus E, Meire F, Aerts P (2010) Low vision affects dynamic stability of gait. Gait Posture 32:547-551. doi:10.1016/J.Gaitpost.2010.07.018

Hartmann M, Grabherr L, Mast FW (2012) Moving along the mental number line: interactions between whole-body motion and numerical cognition. J Exp Psychol Hum Percept Perform 38:1416-1427. doi:10.1037/a0026706

Hartmann M, Furrer S, Herzog MH, Merfeld DM, Mast FW (2013) Self-motion perception training: thresholds improve in the light but not in the dark. Exp Brain Res 226:231-240. doi:10.1007/ S00221-013-3428-1

Hartmann M, Haller K, Moser I, Hossner E-J, Mast FW (2014) Direction detection thresholds of passive self-motion in artistic gymnasts. Exp Brain Res 232:1249-1258. doi:10.1007/ s00221-014-3841-0

Hesterberg TC, Moore DS, Monaghan S, Clipson A, Epstein R (2005) Bootstrap methods and permutation tests. In: Moore DS, McCabe GP (eds) Introduction to the practice of statistics. WH Freeman \& Co, New York
Hötting K, Röder B (2009) Auditory and auditory-tactile processing in congenitally blind humans. Hear Res 258:165-174. doi:10.1016/J.Heares.2009.07.012

International Council of Ophthalmology (2002) Visual standards. Aspects and ranges of vision loss with emphasis on population surveys. Report prepared for the International Council of Opthalmology at the 29th International Congress of Ophthalmology. Sydney, Australia, April 2002. http://www.icoph.org/downloads/ visualstandardsreport.pdf. Accessed 27 Aug 2014

Karmali F, Lim K, Merfeld DM (2014) Visual and vestibular perceptual thresholds each demonstrate better precision at specific frequencies and also exhibit optimal integration. J Neurophysiol 111:2393-2403. doi:10.1152/Jn.00332.2013

King S, Wang J, Priesol AJ, Lewis RF (2014) Central integration of canal and otolith signals is abnormal in vestibular migraine. Front Neurol 5:233. doi:10.3389/fneur.2014.00233

Kömpf D, Piper H-F (1987) Eye movements and vestibulo-ocular reflex in the blind. J Neurol 234:337-341

Leek MR (2001) Adaptive procedures in psychophysical research. Percept Psychophys 63:1279-1292. doi:10.3758/Bf03194543

Lempert $\mathrm{T}$ et al (2012) Vestibular migraine: diagnostic criteria. J Vestib Res 22:167-172. doi:10.3233/Ves-2012-0453

Lewis RF, Priesol AJ, Nicoucar K, Lim K, Merfeld DM (2011a) Abnormal motion perception in vestibular migraine. Laryngoscope 121:1124-1125. doi:10.1002/Lary.21723

Lewis RF, Priesol AJ, Nicoucar K, Lim K, Merfeld DM (2011b) Dynamic tilt thresholds are reduced in vestibular migraine. $\mathrm{J}$ Vestib Res 21:323-330. doi:10.3233/Ves-2011-0422

Machado ML, Lelong-Boulouard V, Philoxene B, Davis A, Denise P, Besnard S (2014) Vestibular loss promotes procedural response during a spatial task in rats. Hippocampus 24:591-597. doi:10.1002/Hipo.22251

MacNeilage PR, Banks MS, DeAngelis GC, Angelaki DE (2010) Vestibular heading discrimination and sensitivity to linear acceleration in head and world coordinates. J Neurosci 30:9084-9094. doi:10.1523/Jneurosci.1304-10.2010

Martin T et al (2015) Vestibular loss disrupts daily rhythm in rats. J Appl Physiol (1985) 118:310-318. doi:10.1152/ japplphysiol.00811.2014

Mast FW, Merfeld DA, Kosslyn SA (2006) Visual mental imagery during caloric vestibular stimulation. Neuropsychologia 44:101109. doi:10.1016/J.Neuropsychologia.2005.04.005

Merfeld DM (2011) Signal detection theory and vestibular thresholds: I. Basic theory and practical considerations. Exp Brain Res 210:389-405. doi:10.1007/S00221-011-2557-7

Mittelstaedt H (1996) Somatic graviception. Biol Psychol 42:53-74. doi:10.1016/0301-0511(95)05146-5

Niemeyer W, Starlinger I (1981) Do the blind hear better-investigations on auditory processing in congenital or early acquired blindness. II. Central functions. Audiology 20:510-515

Occelli V, Spence C, Zampini M (2013) Auditory, tactile, and audiotactile information processing following visual deprivation. Psychol Bull 139:189-212. doi:10.1037/A0028416

Okada T, Grunfeld E, Shallo-Hoffmann J, Bronstein AM (1999) Vestibular perception of angular velocity in normal subjects and in patients with congenital nystagmus. Brain 122:1293-1303. doi:10.1093/Brain/122.7.1293

Pasqualotto A, Proulx MJ (2012) The role of visual experience for the neural basis of spatial cognition. Neurosci Biobehav Rev 36:1179-1187. doi:10.1016/J.Neubiorev.2012.01.008

Postma A, Zuidhoek S, Noordzij ML, Kappers AML (2008) Haptic orientation perception benefits from visual experience: evidence from early-blind, late-blind, and sighted people. Percept Psychophys 70:1197-1206. doi:10.3758/Pp.70.7.1197

Priesol AJ, Valko Y, Merfeld DM, Lewis RF (2014) Motion perception in patients with idiopathic bilateral vestibular 
hypofunction. Otolaryngol Head Neck Surg 150:1040-1042. doi:10.1177/0194599814526557

Raphan T, Matsuo V, Cohen B (1979) Velocity storage in the vestibulo-ocular reflex arc (VOR). Exp Brain Res 35:229-248

Ray CT, Horvat M, Croce R, Mason RC, Wolf SL (2008) The impact of vision loss on postural stability and balance strategies in individuals with profound vision loss. Gait Posture 28:58-61. doi:10.1016/J.Gaitpost.2007.09.010

Röder B, Rösler F (2003) Memory for environmental sounds in sighted, congenitally blind and late blind adults: evidence for cross-modal compensation. Int J Psychophysiol 50:27-39. doi:10.1016/S0167-8760(03)00122-3

Röder B, Rösler F, Spence C (2004) Early vision impairs tactile perception in the blind. Curr Biol 14:121-124. doi:10.1016/J. Cub.2003.12.054

Rombaux P, Huart C, De Volder AG, Cuevas I, Renier L, Duprez T, Grandin C (2010) Increased olfactory bulb volume and olfactory function in early blind subjects. Neuroreport 21:1069-1073. doi:10.1097/Wnr.0b013e32833fcb8a

Sack RL, Brandes RW, Kendall AR, Lewy AJ (2000) Entrainment of free-running circadian rhythms by melatonin in blind people. $\mathrm{N}$ Engl J Med 343:1070-1077. doi:10.1056/Nejm200010123431503

Sanabria-Bohorquez SM, De Volder AG, Arno P, Sibomana M, Coppens A, Michel C, Veraart C (2001) Decreased benzodiazepine receptor density in the cerebellum of early blind human subjects. Brain Res 888:203-211. doi:10.1016/S0006-8993(00)03049-3

Schautzer F, Hamilton D, Kalla R, Strupp M, Brandt T (2003) Spatial memory deficits in patients with chronic bilateral vestibular failure. Ann NY Acad Sci 1004:316-324. doi:10.1196/ annals.1303.029

Schwenn O, Hundorf I, Moll B, Pitz S, Mann W (2002) Do blind persons have a better sense of smell than normal sighted people? Klin Monatsbl Augenheilkd 219:649-654. doi:10.105 5/s-2002-35167

Seemungal BM, Gunaratne IA, Fleming IO, Gresty MA, Bronstein AM (2004) Perceptual and nystagmic thresholds of vestibular function in yaw. J Vestib Res 14:461-466

Seemungal BM, Glasauer S, Gresty MA, Bronstein AM (2007) Vestibular perception and navigation in the congenitally blind. $\mathrm{J}$ Neurophysiol 97:4341-4356. doi:10.1152/Jn.01321.2006

Sherman KR, Keller EL (1986) Vestibulo-ocular reflexes of adventitiously and congenitally blind adults. Invest Ophthalmol Vis Sci 27:1154-1159
Soyka F, Giordano PR, Beykirch K, Bulthoff HH (2011) Predicting direction detection thresholds for arbitrary translational acceleration profiles in the horizontal plane. Exp Brain Res 209:95-107. doi:10.1007/S00221-010-2523-9

Starlinger I, Niemeyer W (1981) Do the blind hear better-investigations on auditory processing in congenital or early acquired blindness. 1. Peripheral functions. Audiology 20:503-509

Sterr A, Green L, Elbert T (2003) Blind Braille readers mislocate tactile stimuli. Biol Psychol 63:117-127. doi:10.1016/ S0301-0511(03)00051-6

Stevens AA, Weaver K (2005) Auditory perceptual consolidation in early-onset blindness. Neuropsychologia 43:1901-1910. doi:10.1016/J.Neuropsychologia.2005.03.007

Taylor MM, Creelman CD (1967) Pest-efficient estimates on probability functions. J Acoust Soc Am 41:782. doi:10.1121/1.1910407

Ungar S, Blades M, Spencer C (1997) Teaching visually impaired children to make distance judgments from a tactile map. J Vis Impair Blind 91:163-174

Valko Y, Lewis RF, Priesol AJ, Merfeld DM (2012) Vestibular labyrinth contributions to human whole-body motion discrimination. J Neurosci 32:13537-13542. doi:10.1523/ Jneurosci.2157-12.2012

Vecchi T, Tinti C, Cornoldi C (2004) Spatial memory and integration processes in congenital blindness. Neuroreport 15:2787-2790

Voss P, Lassonde M, Gougoux F, Fortin M, Guillemot JP, Lepore F (2004) Early- and late-onset blind individuals show supra-normal auditory abilities in far-space. Curr Biol 14:1734-1738. doi:10.1016/J.Cub.2004.09.051

Wan CY, Wood AG, Reutens DC, Wilson SJ (2010) Early but not lateblindness leads to enhanced auditory perception. Neuropsychologia 48:344-348. doi:10.1016/j.neuropsychologia.2009.08.016

Wearne S, Raphan T, Cohen B (1998) Control of spatial orientation of the angular vestibuloocular reflex by the nodulus and uvula. J Neurophysiol 79:2690-2715

World Health Organization (2013) ICD update and revision platform: change the definition of blindness. http://www.who.int/blindness/en/index.html. Accessed 27 Aug 2014

Yates JT, Johnson R, Starz W (1972) Loudness perception of the blind. Audiology 11:368-376

Zwiers MP, Van Opstal AJ, Cruysberg JRM (2001) A spatial hearing deficit in early-blind humans. J Neurosci 21:art. no.-RC142 